\title{
Characterization of a Novel Elliptically Polarized Wiggler
}

K.J. Randalla , Z. Xua , E. Gluskin", I. McNulty ${ }^{a}$, R. Dejus ${ }^{a}$, S. Krinsky ${ }^{b}$, O. Singh ${ }^{b}$, C.-C. Kao ${ }^{b}$, E.D. Johnson $^{\text {b }}$, C.T. Chen ${ }^{c \ddagger}$, G. Meigse

${ }^{a}$ Advanced Photon Source, Argonne National Laboratory, Argonne, Il 60439

bational Synchrotron Light Source, Brookhaven National Laboratory, Upton, NY 11973

'AT\&T Bell Laboratories, 600 Mountain Ave., Murray Hill, NJ 07974

We report on the initial characterization of an elliptically polarized wiggler built by a collaboration between the Advanced Photon Source, the Institute of Nuclear Physics, and the National Synchrotron Light Source. The wiggler has recently been installed in the X13 straight section of the NSLS X-Ray ring. The device consists of a vertical permanent-magnet wiggler and a horizontal electromagnet wiggler. The electromagnets allow the wiggler to produce on-axis circularly polarized soft $\mathrm{x}$-rays with the capability of $\mathrm{AC}$ modulation of the polarization. The maximum switching frequency that can be reached is $100 \mathrm{~Hz}$.

The degree of circular polarization of the radiation from the wiggler was characterized by making magnetic circular dichroism measurements using the X13A soft $\mathrm{x}$-ray beamline. For a vertical deflection parameter, $\mathrm{K}_{\mathrm{x}}$, of 1.6, the dichroism effects at the $\mathrm{Fe}_{2,3}$ edges indicate a degree of circular polarization of approximately $75 \%$ using an aperture with a vertical acceptance of $87 \mu \mathrm{rad}$. Because both the left- and right-circularly polarized photons traverse identical paths through the beamline optical system, significantly smaller intensity fluctuations associated with switching of the polarization were observed than are typically possible at bending magnet beamlines. This, together with the relatively fast modulation rate, means that it will now be feasible to perform a variety of experiments that have not been possible using bending magnet sources.

\section{INTRODUCTION}

Circularly polarized VUV and soft $\mathrm{x}$-ray beams have been used for many years as experimental probes in a wide variety of scientific disciplines covering the biological, chemical and physical sciences. More recently, there has been increasing demand for circularly polarized soft $\mathrm{x}$-ray beams, particularly in the energy region from $500 \mathrm{eV}$ to $2000 \mathrm{eV}$ covering the important $\mathrm{L}_{2,3}$ and $\mathrm{M}_{4,5}$ transitions of the transition metals and rare earth magnetic materials, respectively. Because optical schemes for manipulation of the beam polarization have not been demonstrated over this energy region, one has little choice but to use a source of variable polarization. The first experimental demonstration of soft $\mathrm{x}$-ray magnetic circular dichroism was performed by Chen et al. ${ }^{1}$ In their experiment, bending magnet radiation alternately selected from either above or below the storage ring orbit plane resulted in an elliptically polarized $x$-ray beam at the sample with alternately opposite polarization. Since that time, several soft $\mathrm{x}$-ray beamlines have been built or modified to utilize the off-axis elliptical polarization

Work performed under contracts W-31-109-ENG-38 and DEAC-02-76-CH-00016 of the U.S. Department of Energy.

${ }^{\ddagger}$ Present address : Synchrotron Radiation Research Center, Hsinchu Science-Base Industrial Park, Hsinchu 30077, Taiwan, R.O.C. 


\section{DISCLAMERR}

Portions of this document may be illegible in electronic image products. Images are produced from the best available original document. 
component from a bending magnet. Insertion devices with controllable polarization ${ }^{2}$ would appear to offer significant advantages in this field. We present characterization measurements from such a device.

\section{THE ELLIPTICAL MULTIPOLE WIGGLER}

The elliptical multipole wiggler (EMW) has been built as a collaborative effort between the Advanced Photon Source, the Budker Institute of Nuclear Physics, and the National Synchrotron Light Source (NSLS). ${ }^{3}$ The device, which is installed at the X13 straight section of the NSLS X-Ray ring has 7 vertical permanent magnet poles, 10 horizontal electromagnet poles, and a period of $16 \mathrm{~cm}$. The vertical gap and horizontal electromagnetic field can be varied to tune the vertical and horizontal deflection parameters, respectively. The magnetic fields measured in this device with typical deflection parameters of $\mathrm{K}_{\mathrm{x}}=1.6$ and $\mathrm{K}_{\mathrm{y}}=12$ are shown in figure $1 .^{4}$

One specific advantage of using horizontal electromagnets is that the horizontal magnetic field can be rapidly reversed, thereby reversing the polarization of the on-axis elliptically polarized $\mathrm{x}$ rays. The present device has been designed and successfully tested with switching frequencies up to $100 \mathrm{~Hz}$. Such high frequency operation is clearly impossible with devices relying on mechanical translation of permanent magnet assemblies.

Another attractive feature of the EMW compared with a bending magnet source and some other types of insertion devices is that the elliptically polarized radiation is generated in the orbital plane. In the EMW, an aperture is necessary to remove the two out-of-plane linearly polarized components having vertical deflection angles of $\mathrm{K}_{\mathrm{x}} / \gamma$. Reversal of the onaxis $\mathrm{x}$-ray beam ellipticity is achieved by reversing the EMW horizontal field direction. This means that an identical optical path is traversed for both polarization components. In out-of-plane configurations this is typically not the case, which can result in intensity differences of several percent between the two components.
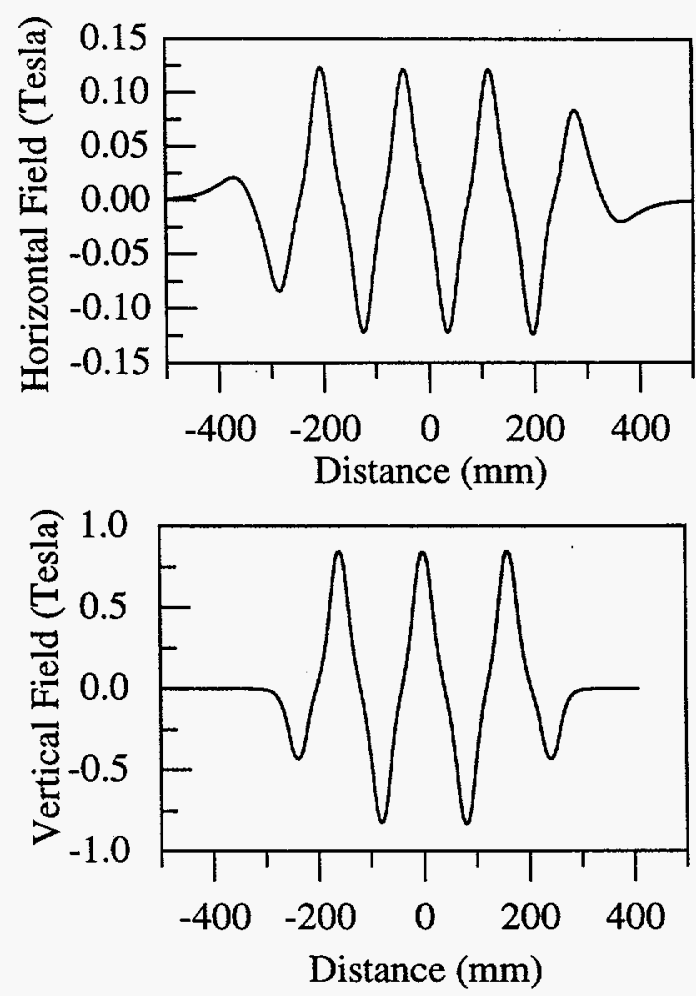

Figure 1. EMW horizontal and vertical magnetic fields ${ }^{4}$

\section{MEASUREMENTS}

The measurements presented here were taken with the EMW and the X13A soft X-ray beamline. ${ }^{5} \mathrm{~A}$ spherical grating monochromator covering the energy range from 300 to $1200 \mathrm{eV}$ was used to monochromatize the wiggler radiation. The grazing incidence optics of this beamline ensure that the source polarization is fully preserved at the sample. The vertical gap of the EMW was set to give a deflection parameter $\mathrm{K}_{\mathrm{y}}$ of 11.5 . The electromagnet current was adjusted to $400 \mathrm{~A}$, corresponding to a $\mathrm{K}_{\mathrm{x}}$ of 1.1 , with a switching frequency of $2 \mathrm{~Hz}$. The monochromatized beam was passed through an aperture in front of the sample. The aperture was mounted on a linear actuator for alignment with the center of the beam. The aperture could also be scanned for beam profile measurements. The aperture, located $23 \mathrm{~m}$ from the source, is $2 \mathrm{~mm}$ 
high, corresponding to a vertical acceptance of 87 $\mu \mathrm{rad}$. The horizontal acceptance of the beamline was limited to approximately $0.5 \mathrm{mrad}$ by a horizontally deflecting mirror.

The circular polarization of the monochromatized beam was measured by taking magnetic circulardichroism measurements of thin magnetic films. The films were deposited on GaAs wafers mounted on a manipulator in a UHV sample chamber. A permanent magnet generated a field of up to $\sim 0.5$ Tesla at the sample surface. The photoemission current from the sample was measured as a function of photon energy. The signal from the sample was synchronized with the switching of the EMW field, digitized, and fed into separate "left-polarization" and "right-polarization" counters .

As an example from our measurements, the MCD signal taken at the $\mathrm{Fe} \mathrm{L}_{2,3}$ edge is shown in figure 2 . This spectrum is shown without normalization to the beam intensity or correction of the beam incidence angle of $45^{\circ}$. The characteristic MCD spectrum indicates a maximum dichroic signal of approximately $14 \%$ at the $L_{3}$ edge, at $c a .708 \mathrm{eV}$.

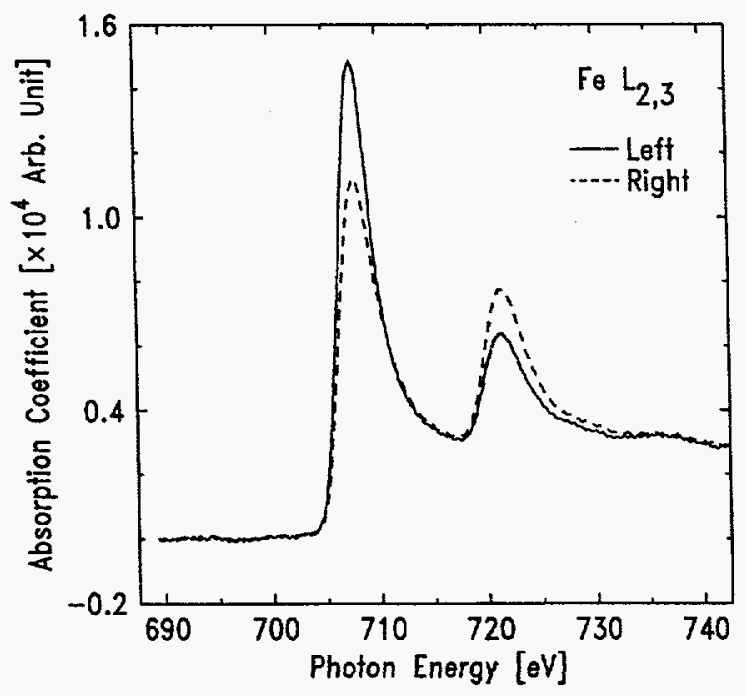

Figure 2. MCD measurement at the $\mathrm{Fe} \mathrm{L}_{2,3}$ edge

The difference in intensity of the left and right channels (as measured on a separate $\mathrm{I}_{0}$ mesh in front of the sample) was measured to be less than $1 \%$ in all cases. However, we noted that the difference was not constant but had an apparent linear energy dependence. We also observed small periodic oscillations of the $I_{0}$, which can be seen at approximately 694,700 , and $738 \mathrm{eV}$ in figure 2 . By normalizing to the $\mathrm{I}_{0}$ signal, the data can be corrected to remove both the linear and oscillating background from figure 2.

In addition to the $I_{0}$ measuremnts, we also noted an unexpected intensity distribution from the EMW when viewing the beam on a fluorescent screen upstream of the monochromator. With no vertical aperture present, one observes two bright horizontal lines, one above and the other below the orbit plane, separated by a vertical deflection angle of $\mathrm{K}_{\mathrm{x}} / \gamma$ as expected. In addition, we observed that one of the lines was brighter than the other and that the pattern oscillated in synchronization with the electromagnetic field.

\section{DISCUSSION}

Comparison of the MCD measurements reported here with data ${ }^{6}$ taken at NSLS Dragon Beamline, U4B, indicates that the wiggler radiation has a circular polarization rate, $S_{3}$, of between 60 and $65 \%$ at the $\mathrm{Fe}_{3}$ edge with $\mathrm{K}_{\mathrm{x}}=1.1$. Results taken at $\mathrm{K}_{\mathrm{x}}=1.6$ show that $\mathrm{S}_{3}$ increases to aproximately $75 \%$. This agrees well with the calculated results shown in figure 3.

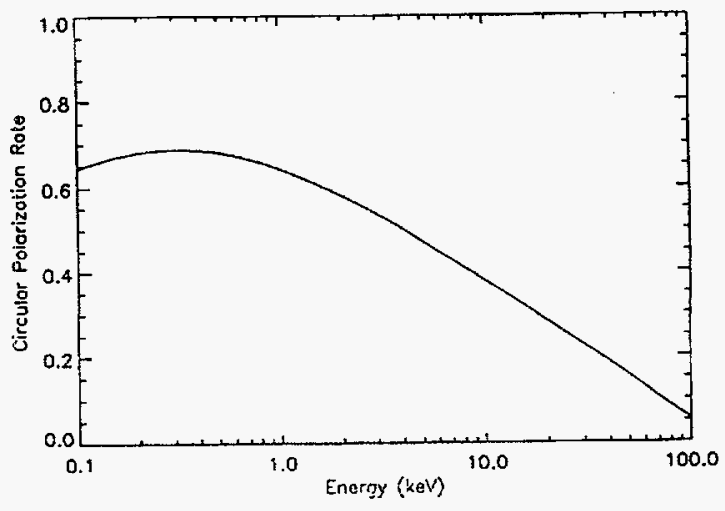

Figure 3. Calculated circular polarization of the EMW 
Our current understanding of the asymmetric vertical intensity distribution is that it is the result of the symetric (w.r.t. reflection in vertical axis through the center of the device) vertical magnetic field (figure 1). This configuration was chosen because it is then easier to compensate the asymmetric, time-dependent horizontal field. Although the magnetic lattice was carefully adjusted to have a negligible vertical field integral, the radiation characteristics of the two halfstrength end poles will differ from those of the full poles. This can be qualitatively explained by approximating each of the vertical poles by bend magnet sources. In this approximation, the end poles have a critical energy of half that of the full poles. Because the vertical angular distribution of radiation from a bending magnet is a function of the photon energy and also depends on the critical energy, we expect the asymmetry of the vertical field to be evident as an energy-dependent asymmetry in the vertical angular distribution.

The oscillations in the background signal are thought to be due to undulator-like interference effects in the radiated field, as qualitatively seen in calculations. Future study periods will be dedicated to investigating this effect.

\section{CONCLUSION}

The EMW behaves largely as predicted. The in-plane production of elliptically polarized radiation results in intensity differences of less than $1 \%$. This will now make it possible to perform measurements on extremely dilute or weakly coupled systems that would otherwise be extremely difficult in out-ofplane configurations. By changing the symmetry of the vertical magnetic field, we believe that the small intensity differences will be reduced further. We hope that our characterization of the EMW will be extremely useful to designers of future insertion devices of controllable polarization.

\section{REFERENCES}

1.C.T. Chen, F. Sette, Y. Ma, and S. Modesti, Phys Rev B 427262 (1990).

2. see for example, P. Elleaume, Rev Sci Instr 60 1830 (1989).

3. E. Gluskin, D. Frachon, P. Ivanov, J. Maines, E. Medvedko, E. Trakhtenberg, L. Turner, I. Vasserman, N. Gavrilov, Y. Evtuchenko, G. Erg, G. Kulipanov, A. Medvedko, S. Petrov, V. Popik, N. Vinokurov, A. Friedman, S. Krinsky, G. Rakowsky, and O. Singh, Proceedings of 1995 Particle Accelerator Conference (May 1-5, 1995, Dallas, Texas).

4. D. Frachon, P.M. Ivanov, E.A. Medvedko, I. Vasserman, O. Despe, Y.G. Kang, Proceedings of 1995 Particle Accelerator Conference (May 1-5, 1995, Dallas, Texas).

5. Z. Xu, I. McNulty, K.J. Randall, L. Yang, E. Gluskin, E.D. Johnson, and T. Oversluizen, Rev. Sci. Instrum. 661827 (1995).

6. C.T. Chen, Rev. Sci. Instr. 631229 (1992).

\section{DISCLAIMER}

This report was prepared as an account of work sponsored by an agency of the United States Government. Neither the United States Government nor any agency thereof, nor any of their employees, makes any warranty, express or implied, or assumes any legal liability or responsibility for the accuracy, completeness, or usefulness of any information, apparatus, product, or process disclosed, or represents that its use would not infringe privately owned rights. Reference herein to any specific commercial product, process, or service by trade name, trademark, manufacturer, or otherwise does not necessarily constitute or imply its endorsement, recommendation, or favoring by the United States Government or any agency thereof. The views and opinions of authors expressed herein do not necessarily state or reflect those of the United States Government or any agency thereof. 